\title{
Uma estratégia de integração entre plantas baixas CAD e Realidade Virtual: estudo de caso aplicado a subestações de energia elétrica
}

An integration strategy between CAD floor plans and Virtual Reality: case study applied to electric power substations

Una estrategia de integración entre planos CAD y Realidad Virtual: estudio de caso aplicado a subestaciones de energía eléctrica

Camilo de Lellis Barreto Junior ORCID: https://orcid.org/0000-0001-7250-605X

Universidade Federal de Uberlândia, Brasil E-mail: camilobarreto@ufu.br Paulo Camargos Silva ORCID: https://orcid.org/0000-0002-3542-7368 Universidade Federal de Uberlândia, Brasil E-mail: paulo.camargos@ufu.br

Alexandre Carvalho Silva ORCID: https://orcid.org/0000-0003-0264-3475 Instituto Federal Goiano, Brasil E-mail: alexandre.silva@ifgoiano.edu.br

Edgard Afonso Lamounier Junior ORCID: https://orcid.org/0000-0001-6293-9521

Universidade Federal de Uberlândia, Brasil E-mail: lamounier@ufu.br

Alexandre Cardoso

ORCID: https://orcid.org/0000-0002-2023-9647

Universidade Federal de Uberlândia, Brasil E-mail: alexandre@ufu.br

\begin{abstract}
Resumo
Muitos setores têm se beneficiado com a tecnologia de Realidade Virtual (RV), como, por exemplo, as indústrias na aplicação em processos de revisão de desenhos de engenharia. É amplamente atribuída ao treinamento de operadores, arquitetura, engenharia, educação, simulação e prototipagem, pois proporciona um alto nível de imersão e fidelidade na representação das informações. O contexto atual sobre a integração entre Computer-Aided Design (CAD) e RV apresenta métodos de desenvolvimento e manutenção demasiadamente complexos, exigindo intervenções manuais que dificultam a transferência das informações, expressando um estado imaturo da tecnologia. Diante deste cenário, o trabalho propõe uma metodologia de integração entre plantas baixas CAD e RV através de uma arquitetura de software que possibilita realizar a união entre as tecnologias de forma automática. Foi realizada uma análise em trabalhos correlatos com a finalidade de obter o estado da arte e as tecnologias que estão sendo utilizadas, além de apontar a contribuição da metodologia proposta. Através de um estudo de caso aplicado a subestações de energia elétrica em um projeto de Pesquisa e Desenvolvimento (P\&D), foi realizado testes de integração, reconstrução do Ambiente Virtual (AV) e desempenho. A metodologia apresentou excelentes resultados, a integração entre plantas baixas CAD e o software de Realidade Virtual demonstrou ser possível adequar o espaço bidimensional CAD para representação em um AV tridimensional, facilitando e melhorando o processo de concepção de subestações virtuais. Também, o processo de reconstrução apresentou um desempenho satisfatório, concluindo as tarefas em tempos curtos e sem a necessidade de processos manuais de usuários.
\end{abstract}

Palavras-chave: Integração; Computer-Aided Design; Realidade virtual; Arquitetura de Software; Modelagem 3D.

\begin{abstract}
Many sectors have benefited from Virtual Reality (VR) technology, such as industries in the application of engineering design review processes. It is widely used in operator training, architecture, engineering, education, simulation and prototyping, as it provides a high level of immersion and fidelity in the representation of information. The current context of the integration between Computer-Aided Design (CAD) and VR presents development and maintenance methods that are too complex, requiring manual interventions making the transfer of information difficult, expressing an immature state of technology. Given this scenario, this work proposes a methodology for integration of CAD and VR floor plans through a software architecture that makes it possible to process the union
\end{abstract}


between the technologies automatically. An research was conducted for related works in order to obtain the state of the art and the technologies in current use, in addition to pointing out the contribution of the proposed methodology. A case study was applied to electric power substations in a Research and Development (R\&D) project. Integration tests, Virtual Environment (AV) reconstruction and performance tests were executed. The methodology showed good results as with the integration of CAD floor plans and the Virtual Reality software. In this paper, it is demonstrated that it is possible to adapt the two-dimensional CAD space for representation in a three-dimensional AV, facilitating and improving the design process of virtual substations. Also, the reconstruction process showed a satisfactory performance, completing the tasks in shorter times without manual interventions.

Keywords: Integration; Computer-Aided design, Virtual reality; Software architecture; 3D modeling.

\section{Resumen}

Muchos sectores se han beneficiado de la tecnología de Realidad Virtual (RV), como, por ejemplo, las industrias en la aplicación de procesos de revisión de diseños de ingeniería. Se atribuye ampliamente a la formación de operadores, arquitectura, ingeniería, educación, simulación y creación de prototipos, ya que proporciona un alto nivel de inmersión y fidelidad en la representación de la información. El contexto actual de la integración entre el ComputerAided Design (CAD) y la VR presenta métodos de desarrollo y mantenimiento demasiado complejos, que requieren intervenciones manuales que dificultan la transferencia de información, expresando un estado inmaduro de la tecnología. Ante este escenario, el trabajo propone una metodología de integración entre planos CAD y VR a través de una arquitectura de software que permite realizar la unión entre las tecnologías de forma automática. Se realizó un análisis en trabajos relacionados con el fin de obtener el estado del arte y las tecnologías utilizadas, además de señalar el aporte de la metodología propuesta. A través de un caso de estudio aplicado a subestaciones eléctricas en un proyecto de Investigación y Desarrollo (I+D), se realizaron pruebas de integración, reconstrucción de Ambiente Virtual (AV) y pruebas de desempeño. La metodología arrojó excelentes resultados, la integración entre planos CAD y el software de Realidad Virtual demostró que es posible adaptar el espacio CAD bidimensional para su representación en un AV tridimensional, facilitando y mejorando el proceso de diseño de subestaciones virtuales. Asimismo, el proceso de reconstrucción presentó un desempeño satisfactorio, completando las tareas en tiempos cortos y sin necesidad de procesos manuales de usuario.

Palabras clave: Integración; Computer-aided design; Realidad virtual; Arquitectura de software; Modelado 3D.

\section{Introdução}

Muitos setores têm se beneficiado com a tecnologia da Realidade Virtual (RV), como, por exemplo, as indústrias, na aplicação em processos de revisão de desenhos de engenharia (Wolfartsberger, 2019). A RV é uma interface avançada para aplicações computacionais que permite ao usuário navegar e interagir em tempo real em ambientes tridimensionais (3D) utilizando dispositivos multissensoriais para atuação ou feedback (Tori, Kirner, \& Siscoutto, 2006), além de proporcionar um alto nível de imersão e fidelidade na representação das informações (ISO, 2020). A RV é uma das tecnologias emergentes mais importantes da atualidade (Roldán et al., 2019). É amplamente atribuída aos processos de treinamento, testes industriais (Kovar et al., 2016; Kuts et al., 2018), arquitetura, engenharia (Pessoa et al., 2017), educação, simulação e prototipagem rápida (Ciprian Firu et al., 2021).

Estudos sobre a integração do Computer-Aided Design (CAD) em ambientes imersivos com finalidade de avaliar projetos de engenharia tem ganhado notoriedade (Nugraha Bahar et al., 2014). Muitos aspectos dos ambientes imersivos apresentam benefícios, como a navegação, pelos modelos virtuais e a investigação da geometria e suas proporções de uma forma irrealizável em ambientes reais (Stark, Israel, \& Wöhler, 2010, pp. 179-182), além de possibilitar treinamento e aperfeiçoamento da capacidade técnica dos usuários (Quevedo et al., 2017, pp. 185-198).

Trabalhos recentes sobre a integração entre desenhos de engenharia CAD e RV apresentam processos de desenvolvimento e manutenção demasiadamente complexos, envolvendo equipes de programação do sistema, concepção dos cenários 3D e interfaces gráficas de usuário (GUI), expressando um estado imaturo da tecnologia. Um dos fatores que influenciam a integração, são as tarefas manuais, pois independentemente da fonte ou categoria de modelo 3D, muitas vezes eles precisam ser convertidos, otimizados e preparados para uso efetivo no ambiente de RV, criando um processo inapropriado para o usuário final.

Desta forma, diante do cenário de dificuldades apresentado, este trabalho visa desenvolver e avaliar uma metodologia 
de integração entre plantas baixas CAD e RV aplicado ao estudo de caso (EC) de desenhos de engenharia de subestações de energia elétrica (SEE) através de um projeto de Pesquisa e Desenvolvimento (P\&D). Sendo assim, pretende-se simplificar a união destas tecnologias e automatizar o processo através de um Sistema de Integração (SI) utilizando estrutura de dados, metamodelos e uma arquitetura de software baseada no conceito de serviços em rede. Neste sentido, são enumerados os objetivos específicos:

1. Elaborar uma metodologia para integração unidirecional entre desenhos CAD de plantas baixas e software de Realidade Virtual;

2. Elaborar uma arquitetura de software para viabilizar a integração de forma automática e sem a necessidade de intervenção manual;

3. Avaliar a proposta de integração através da planta baixa CAD de uma subestação de energia elétrica e seu Ambiente Virtual (AV) gerado em RV;

4. Avaliar o desempenho da integração e reconstrução através de testes aplicados em diferentes plantas baixas com configurações de elementos semelhantes aos encontrados em SEEs, utilizando computadores com hardwares compatíveis com empresas, residências e escolas.

\section{Trabalhos Correlatos}

Han et al. (2019) propõe o desenvolvimento de uma ferramenta de RV e Realidade Aumentada (RA) para realizar análises e revisões de arranjos de tubulação de estruturas marítimas. A arquitetura do sistema apresenta tecnologias CAD utilizadas no processo de elaboração das tubulações. Segundo os autores, os testes de implementação e do protótipo desenvolvido, evidenciam que a estratégia adotada pode ser aplicada na conversão e integração de softwares CAD para modelos de tubulações, facilitando a utilização em aplicativos de RV/RA sem a necessidade de retoque manual em um software de modelagem 3D.

O trabalho proposto por Freeman et al. (2016), apresenta o desenvolvimento de um sistema de RV que proporciona o carregamento de modelos paramétricos CAD e viabiliza a manipulação de objetos e seus atributos, sem descredibilizar o realismo e as características que possuem originalmente no software CAD. Sendo assim, os autores propõem o desenvolvimento de um conjunto de ferramentas de integração. Os resultados sugerem que o desenvolvimento da solução, permitiu sincronizar software CAD parametrizado e possibilitou a revisão de projetos de engenharia mecânica com RV, tornando-a mais intuitiva e atrativa, além de melhorar a capacidade do usuário de entender a geometria e obter confiança nas tomadas de decisões.

O trabalho de Lorenz et al. (2016) propõe o desenvolvimento de uma metodologia de conversão automatizada de modelos CAD em um formato amplamente utilizado por aplicações de RV e RA, que dispõe de informações adicionais pertinentes à modelos CAD como: dados de simulações, animações e cinemática. Algumas limitações foram evidenciadas, uma em específico é a falta de arquivos compatíveis com CAD e RV. Os autores aplicaram testes para analisar os benefícios da metodologia proposta, e comprovaram que o conceito apresenta um método funcional, capaz de fornecer modelos CAD com animações e cinemáticas a um ambiente de RV, sem a necessidade de processos manuais complexos para tal.

Martin et al. (2017) propõe o desenvolvimento de ferramentas que possibilitam a integração de modelos CAD em sistemas de RV. Os autores apresentam uma metodologia de integração utilizando o conceito de metamodelo, o qual define uma estrutura de dados que reúne diversas informações advindas de diferentes sistemas. Dessa forma, os autores elaboraram um metamodelo retendo informações do sistema de Product Data Management (PDM), modelo 3D, CAD e simulações no formato representado em arquivo Extensible Markup Language (XML). 
Mattioli (2015) propõe o desenvolvimento de ferramentas para integrar o software CAD e Unity (Technologies, [s.d.]). Tal trabalho apresenta uma característica diferente entre os outros, o desenho CAD é bidimensional (2D). A metodologia realiza a extração de informações da planta baixa e realiza a exportação destas informações para o editor de cenários do Unity, onde posteriormente usuários realizam a inserção e posicionamento dos elementos. Os resultados apresentados demonstraram que um cenário construído utilizando a metodologia convencional durou 20 horas para ser concluído, porém, utilizando a metodologia proposta o tempo foi reduzido para 6 horas.

Zawadzki et al. (2018) apresenta um processo integrado de design de produtos configuráveis que inclui dois processos, (i) configuração de uma variedade de produto com a participação do cliente e (ii) preparação de documentação técnica. Os autores propõem o desenvolvimento de uma ferramenta de RV, integrando o conceito de produtos configuráveis aplicado a customização de um ônibus urbano, com recursos para ajustes de arranjos de poltronas, corrimão e outros aspectos internos do produto. Para tal solução optou-se por modelar produtos em CAD com a tecnologia generativa, uma estratégia de criação de modelos contendo regras de modelagem e lógicas que possibilitam desenvolver um modelo primário com componentes modificáveis seguindo regras pré-definidas. Segundo os autores, os testes com o Virtual Design Studio (VDS) confirmam a possibilidade de integração de modelos CAD com sistemas de RV utilizando a metodologia proposta, tendo como vantagem a redução do tempo necessário para a preparação do design e documentação técnica.

\subsection{Análise Comparativa}

Após a leitura e análise dos trabalhos correlatos, foram identificadas características que evidenciam metodologias de integração entre elementos CAD e RV. Além das tecnologias e metodologias apresentadas, foram destacadas outras características desejáveis, como o uso de grafos e informações de topologia dos elementos que compõem o AV. Foram extraídas as seguintes características:

- Categoria de desenho CAD: bidimensional ou tridimensional;

- Método de integração: manual, semiautomático (envolve tarefas manuais) e automático;

- Metamodelo: abstração do sistema que descreve os aspectos estruturais e/ou comportamentais do software (Guedes, 2012), é uma definição de estrutura de dados;

- Grafos: estrutura de dados que define a hierárquica dos elementos do modelo/cenário;

- Topologia: está relacionada com os grafos. Define os relacionamentos lógico entre elementos do desenho;

- Reconstrução em tempo de execução (RTE): o SRV realiza a reconstrução do AV durante sua inicialização é mais suscetível a atualização de forma rápida.

A partir das metodologias de integração extraídas dos trabalhos, a Tabela 1 foi elaborada. 
Tabela 1. Principais características extraídas das metodologias de integração apresentadas nos trabalhos correlatos.

\begin{tabular}{lllllll}
\hline Trabalho & CAD & Integração & Metamodelo & Grafos & Topologia & RTE \\
\hline Han et al. (2019) & 3D & Automático & Não & Não & Não & Sim \\
\hline Freeman et al. (2016) & 3D & Automático & Não & Não & Não & Sim \\
\hline Lorenz et al. (2016) & 3D & Automático & Não & Não & Não & Sim \\
\hline Martin et al. (2017) & 3D & Automático & Sim & Não & Não & Não \\
\hline Mattioli (2015) & 2D & Semiautomático & Não & Não & Não & Não \\
\hline Zawadzki et al. (2018) & 3D & Semiautomático & Não & Não & Não & Não \\
\hline
\end{tabular}

Fonte: Autores.

Os trabalhos abordaram desenhos CAD de engenharia com representação espacial bidimensional e tridimensional, cada uma é relacionada a sua natureza e proposta de projeto. Independente da representação, os trabalhos apresentaram estratégias de integração entre CAD e RV, evidenciando os resultados e limitações.

A utilização de metamodelos, grafos e topologias são referentes as estruturas de dados utilizadas para a integração entre às duas tecnologias. Nenhum trabalho utilizou simultaneamente essas estruturas, evidenciando um ponto forte a ser estudado.

O método de integração é um aspecto importante em sistemas de RV, pois se espera que o processo seja automático, sem intervenção manual, aprimorando a usabilidade por usuários não especializados na tarefa de integração. Os trabalhos Mattioli, (2015) e Zawadzki et al. (2018) apresentaram a forma semiautomática, necessitando em determinados momentos o trabalho manual de integração.

Mesmo apresentando contextos de aplicações distintos nas engenharias, foi possível evidenciar nos trabalhos citados, a importância do desenvolvimento de mecanismos de integração entre desenhos de engenharia CAD e sistema de RV.

\section{Metodologia}

Nesta seção são apresentados os conceitos e especificações da abordagem proposta neste trabalho. Inicialmente é apresentada a arquitetura de software do SI e o detalhamento de seus componentes. Em seguida, é apresentada a estratégia de integração com o SRV.

\subsection{Arquitetura de Software do Sistema de Integração}

A arquitetura do SI é baseada no conceito cliente-servidor. A Figura 1 apresenta o diagrama conceitual da arquitetura. Ela é composta por três softwares: Editor CAD (ECAD); Gerenciador de Integração (GI) e o software de RV. Todos os componentes trabalham independentemente, porém compartilhando informações através do GI. 
Figura 1. Arquitetura de software do SI.

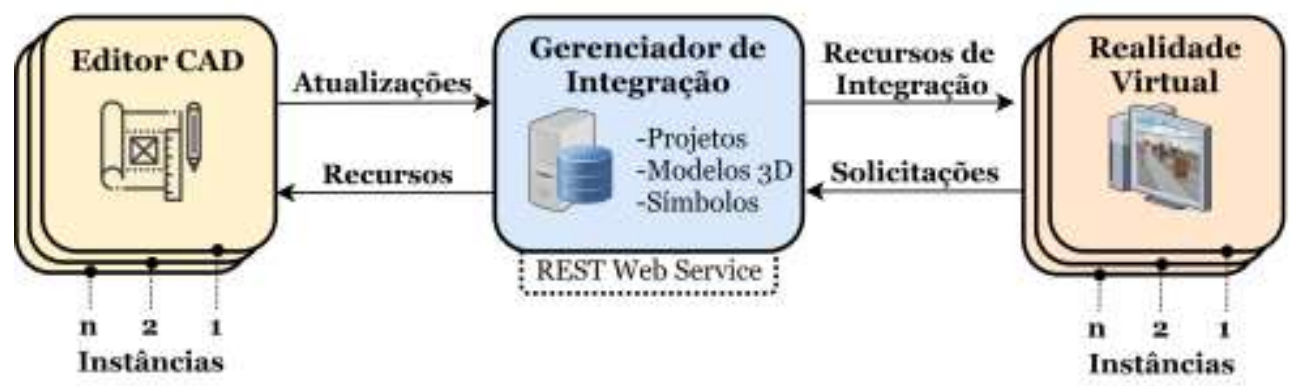

Fonte: Autores.

O ECAD é composto por ferramentas integradas ao software AutoCAD que auxilia o projetista a elaborar as plantas baixas a partir dos itens cadastrados no GI, o qual oferece recursos como (i) abrir e atualizar projetos do usuário; (ii) pesquisar, inserir, editar e substituir símbolos que representam um modelo 3D; (iii) criar símbolos CAD e relacionar com modelos 3D; (iv) inserir e editar condutores elétricos; (v) definir altura de elementos acima do solo e (vi) compilar e integrar a planta baixa no SRV. O ECAD foi implementado no formato add-on (plugin) utilizando a Application Programming Interface (API) fornecida pelo software AutoCAD. Foi projetada para que usuários possam trabalhar independentemente em projetos distintos, possibilitando a concepção e edição de desenhos CAD paralelamente.

O GI é um software de rede responsável por armazenar as informações de projetos CAD e prover serviços para aplicações externas através de um serviço de rede do tipo Representational State Transfer (REST). O software armazena e gerencia os modelos 3D, símbolos $\mathrm{CAD}$, lista de projetos de plantas baixas, versões históricas projetos, gerenciamento de usuários e documentos. O GI mantém uma base de dados relacional entre modelos 3D, símbolos, subestações, versionamento de projeto, informações dos elementos (tensão, fabricante, classe de objeto e outros) e possui uma interface gráfica amigável e de fácil uso.

O SRV é destinado ao usuário final, para realizar tarefas no Ambiente Virtual 3D. O SRV foi projetado para ser utilizado paralelamente, possibilitando usuários acessarem o mesmo AV simultaneamente. Desta forma, o SRV realiza o acesso ao arquivo de integração por meio do GI, efetua a transferência dos modelos necessários para o AV e reconstrói o cenário 3D em um curto processo de tempo.

A comunicação entre os softwares é realizada através de requisições Hypertext Transfer Protocol (HTTP) enviadas ao serviço de rede do GI. Todos os dados são serializados e enviados em pacotes com formato JavaScript Object Notation (JSON) ao requisitante.

Os dados consumidos pelo ECAD são projetos em planta baixa, símbolos CAD e as informações sobre símbolos (identificação, classe, tensão, fabricante, modelo 3D). Em eventuais edições no desenho o usuário pode solicitar a atualização no das informações no GI.

Da mesma forma, o SRV envia requisições ao GI solicitando o arquivo de integração de determinado desenho de engenharia. Uma vez recebido, o arquivo é utilizado para a reconstrução do AV no SRV.

\subsection{Desenho da Planta Baixa}

O desenho da planta baixa é realizado utilizando as ferramentas do ECAD e do software AutoCAD para manipular os símbolos. Os símbolos são representações bidimensionais dos modelos 3D armazenados no GI. 
Neste sentido, os modelos 3D estão armazenados no GI e os símbolos são desenhados e atribuídos a eles. A ferramenta de criação de símbolos solicita ao usuário a seleção do símbolo a ser cadastrado, em seguida, a indicação do modelo 3D a ser atribuído. Uma requisição do tipo HTTP/POST é enviada ao GI por meio da Uniform Resource Locator (URL) "http://servidor:porta/api/symbols/" carregando no pacote as seguintes informações:

- Block: símbolo CAD no formato base 64 para compartilhamento via internet;

- Thumbnail: miniatura rasterizada do símbolo;

- Description: descrição do elemento;

- Objects 3D: código de identificação do modelo 3D que o símbolo foi atribuído.

A inserção dos símbolos na planta baixa é realizada por uma ferramenta implementada com funcionalidades para busca, seleção e inserção de elementos. Primeiramente, o usuário realiza a busca através de filtros informando a classe, descrição ou código do elemento. Os serviços do GI retornam uma lista de símbolos apresentando imagens em miniaturas e suas informações. Ao selecionar o símbolo desejado e executar o processo de inserção, uma requisição HTTP/GET é enviada ao GI por meio da URL "http://servidor:porta/api/symbols/?id=1", onde o trecho "id=1" representa a identificação do elemento selecionado. Logo em seguida, o ECAD recebe o pacote de dados solicitado, contendo informações do símbolo e do modelo 3D:

- Fingerprint: identificação global do símbolo na base de dados do GI;

- Thumbnail: miniatura rasterizada do símbolo;

- Block: símbolo CAD no formato base 64 para serialização dos dados via internet;

- Description: descrição do elemento;

- Object 3D: código de identificação do modelo 3D que o símbolo representa.

Com o pacote de dados na memória do software, o processo de conversão do código em base 64 do elemento "block" para um símbolo CAD é executado. Em seguida, o símbolo é apresentado na viewport da planta baixa e solicitado ao usuário que forneça o valor de rotação (em graus) e a posição no plano para sua inserção. A ferramenta de inserção realiza uma injeção de atributos no símbolo, como apresentado na Figura 2, referente a um equipamento elétrico. As informações atribuídas são metadados importantes para o processo de integração, descrito a seguir. Metadados é definido como dados sobre outros dados, são conjuntos de informações para outra categoria de informação (Baca \& Getty Research Institute, 2008).

\subsection{Processo de Integração}

A estratégia proposta para a integração entre o software ECAD e SRV é o uso de metadados, estruturas de dados hierárquicos e conceito de metamodelo. Por se tratarem de softwares com espaços dimensionais diferentes, há o desafio de recriar o que está no plano 2D em 3D, sem a necessidade de ações manuais.

A Figura 2 apresenta o diagrama conceitual do processo de extração e adequação dos dados para integração entre ECAD e SRV. Na etapa de manipulação da planta baixa, os símbolos são obtidos por meio do GI e inseridos no desenho, atribuindo informações do modelo 3D relacionado. Posteriormente, quando solicitado pelo usuário, o processo de integração é executado e realizado em três etapas: 
- Extração da estrutura dos grafos: o processo obtém todos os relacionamentos entre símbolos de forma hierárquica e armazena temporariamente em uma estrutura de dados na memória;

- Extração dos metadados: o processo extrai os atributos dos símbolos inseridos durante a concepção do desenho. Também é extraído informações adicionais como a matriz de transformação (posição, rotação e escala);

- Aplicação do metamodelo: o processo organiza a estrutura de grafos e metadados em uma estrutura de dados unificada. Nessa etapa, os dados são organizados em blocos e mantendo a hierarquia dos elementos. Todas as informações são armazenadas em um arquivo com formato XML, para possibilitar o compartilhamento de dados pela internet.

Figura 2. Diagrama conceitual do processo de integração a partir do ECAD.

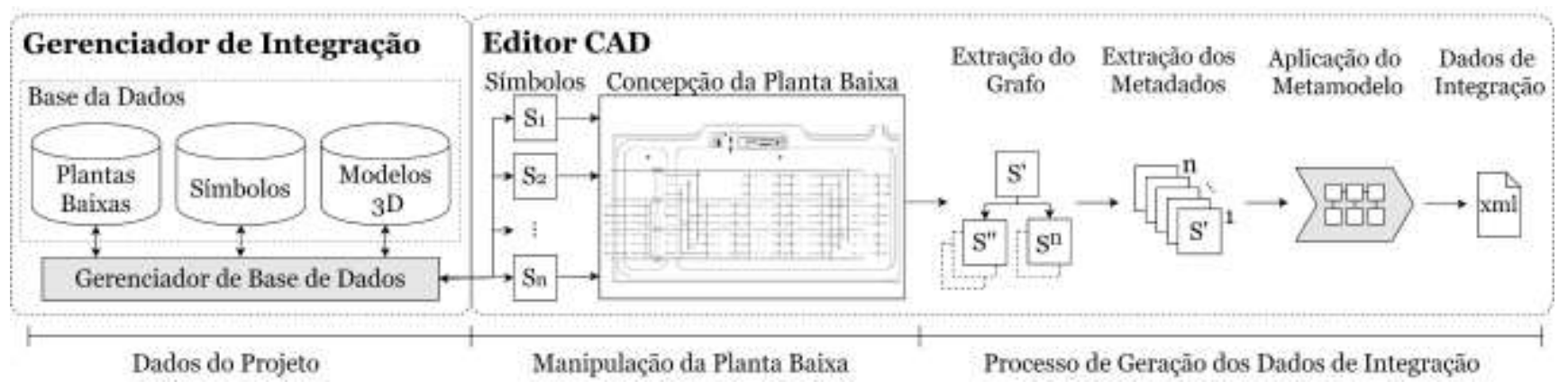

Fonte: Autores.

A Figura 3 apresenta um diagrama conceitual do processo de recuperação pelo SRV do arquivo de integração, extração dos dados, transferência e atualização dos modelos 3D locais e a reconstrução do ambiente virtual em RV.

Figura 3. Diagrama conceitual do processo de integração a partir do software de Realidade Virtual.

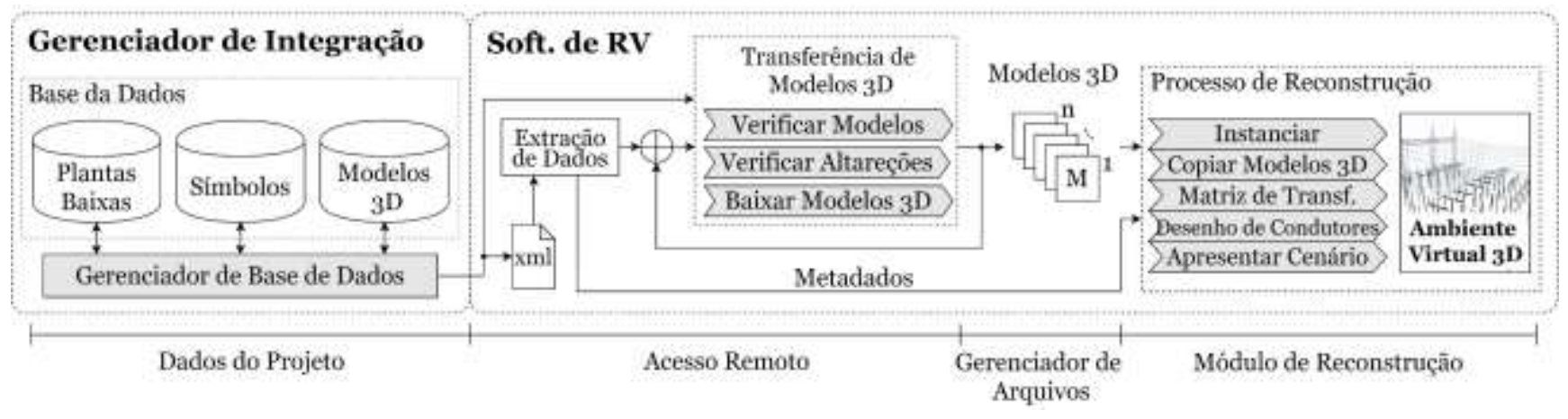

Fonte: Autores.

Através de algoritmos de extração de metamodelo, o arquivo é decomposto e os dados são utilizados para realizar a transferência e atualização de modelos, bem como para a reconstrução do cenário 3D.

A transferência de modelos $3 \mathrm{D}$ é realizada em três etapas. Na primeira etapa é realizada uma verificação no diretório local para obter os modelos baixados. Na segunda os modelos $3 \mathrm{D}$ do diretório local são comparados com os modelos armazenados no GI e caso haja diferença entre eles, os modelos locais são atualizados. Por fim, na terceira etapa é realizada a transferência dos modelos não presentes no diretório local. O processo é realizado antes da reconstrução do cenário, garantindo o acesso a todos os modelos necessários para a reconstrução. 
Após a transferência dos modelos 3D, o processo de reconstrução é iniciado. Ele é executado em quatro etapas:

1. Instanciar modelos 3D: todos os modelos são instanciados na memória. Esse processo é oneroso em questão de processamento, sendo executado apenas uma vez para novos elementos;

2. Copiar modelos 3D: a partir dos modelos 3D instanciados na memória o SRV realiza cópias para o AV;

3. Aplicar matriz de transformação: é aplicado as informações de posição, rotação e escala de cada elemento;

4. Desenho de condutores: os cabos e barramentos rígidos de condução de eletricidade são desenhados de forma automática. Este processo usa os dados de topologia configurados durante a confecção da planta baixa;

5. Apresentar cenário: durante os processos anteriores nenhuma informação visual é apresentada ao usuário, somente quando o processo de reconstrução é finalizado o software apresenta o cenário ao usuário.

\subsection{Desenho dos Condutores}

Diferentemente dos modelos 3D armazenados no GI que são transferidos, carregados na memória e inseridos no AV, os condutores são elementos construídos em tempo de execução, isso é, eles são desenhados por algoritmos que concebe as malhas dos condutores durante o processo de reconstrução e se ajustam às distâncias entre os elementos.

Os modelos 3D possuem ancoragens denominadas "conectores" utilizados para referenciar o local de conexão do condutor. Há diferentes quantidades de condutores em diferentes tipos de ativos, contendo no mínimo dois conectores (entrada e saída da linha). A Figura 4 apresenta os pontos de conexão de cabos no "Conector A" e "Conector B".

Figura 4. Conectores de um equipamento elétrico.

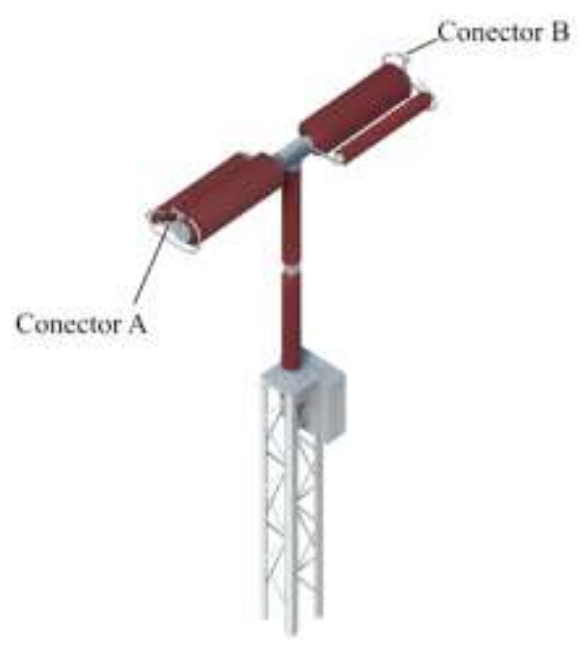

Fonte: Autores.

O Algoritmo 1 apresenta como os conectores são selecionados entre equipamentos e como é desenhado os condutores. Quando equipamentos possuem mais de dois conectores é necessário definir a quantidade de cabos interligados entre os ativos. Para isso, foi incluído no algoritmo um fator que delimita a quantidade de conexões de cabos que serão realizados. 


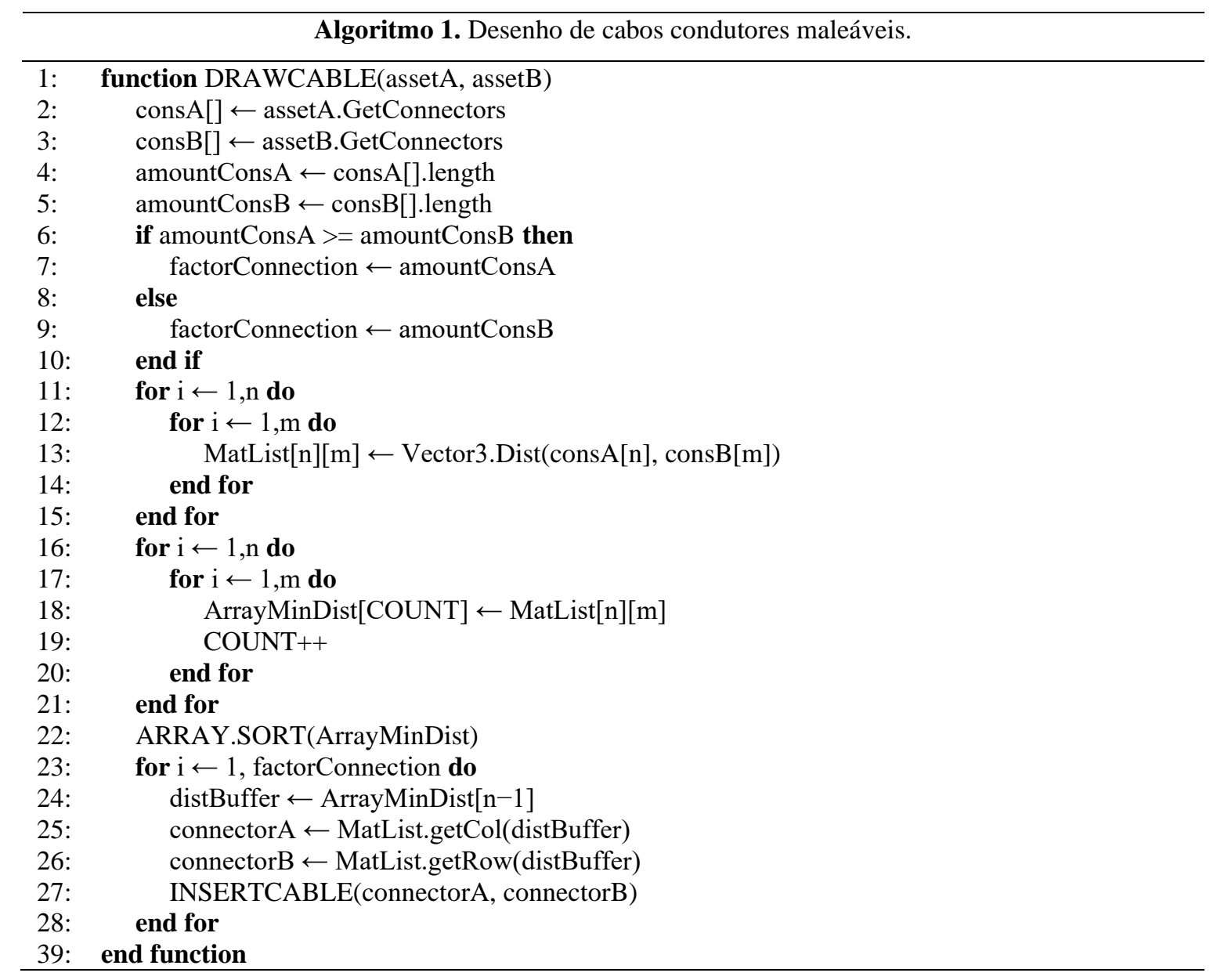

Fonte: Autores.

Um exemplo do fator de conexão, é uma dada chave seccionadora que possui 2 conectores ao ser conectada com um disjuntor que possui 4, o fator de conexão considerado é de 1 para 2. Isso significa que a chave seccionadora possui dois cabos conectados a um conector, por outro lado, o disjuntor tem dois cabos, cada um interligado a um conector. A Figura 5 demonstra este cenário.

Figura 5. Conexão via cabo maleável entre dois equipamentos.

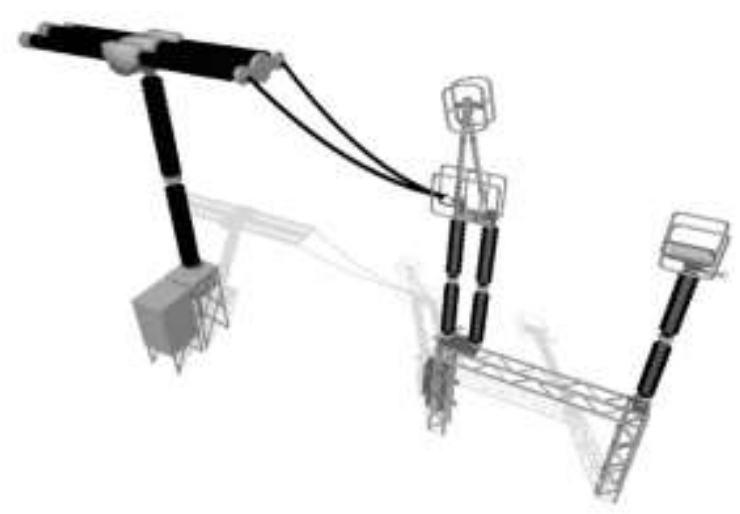

Fonte: Autores. 


\section{Resultados da Avaliação Experimental}

A avaliação experimental foi realizada através de um estudo de caso aplicado ao projeto de P\&D de uma companhia de energia elétrica com abrangência no fornecimento de eletricidade para o Brasil. O projeto permeia temas de pesquisa em RV para supervisão e treinamento para sistemas críticos de energia elétrica, além de interfaces homem-computador que viabilizam os três pilares da RV: interação, imersão e imaginação (Sheridan, 2000).

Para o EC e avaliação da proposta, foi realizada a concepção de uma planta baixa de uma SEE que opera com o nível de tensão de 345 mil volts em uma área de cerca de treze mil metros quadrados. A Figura 6 apresenta a planta baixa desenvolvida utilizando o ECAD. Há 312 elementos no total, incluindo estruturas, equipamentos e construções.

Figura 6. Planta baixa desenvolvida utilizando o ECAD e os recursos do GI.

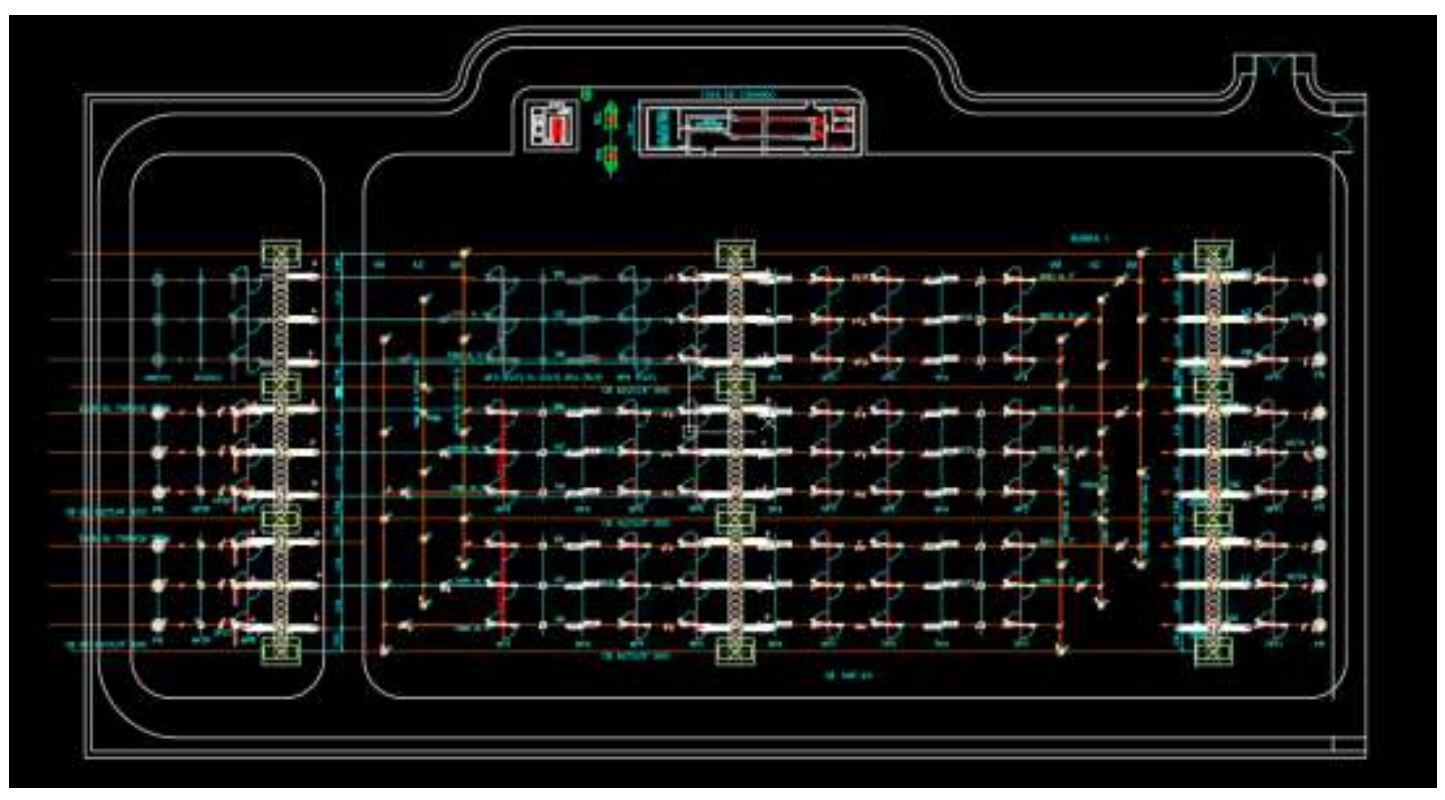

Fonte: Autores.

A modelagem 3D e de símbolos foi realizada utilizado o software 3Ds Max. A Figura 7 demonstra alguns elementos modelados e utilizados na SEE. Os modelos 3D foram concebidos utilizando como referência documentos CAD, fotografias e cortes laterais, obtendo grande credibilidade em relação ao elemento real.

Figura 7. Modelos 3D e símbolos da subestação elétrica construídos utilizando o 3Ds Max e o Editor CAD.

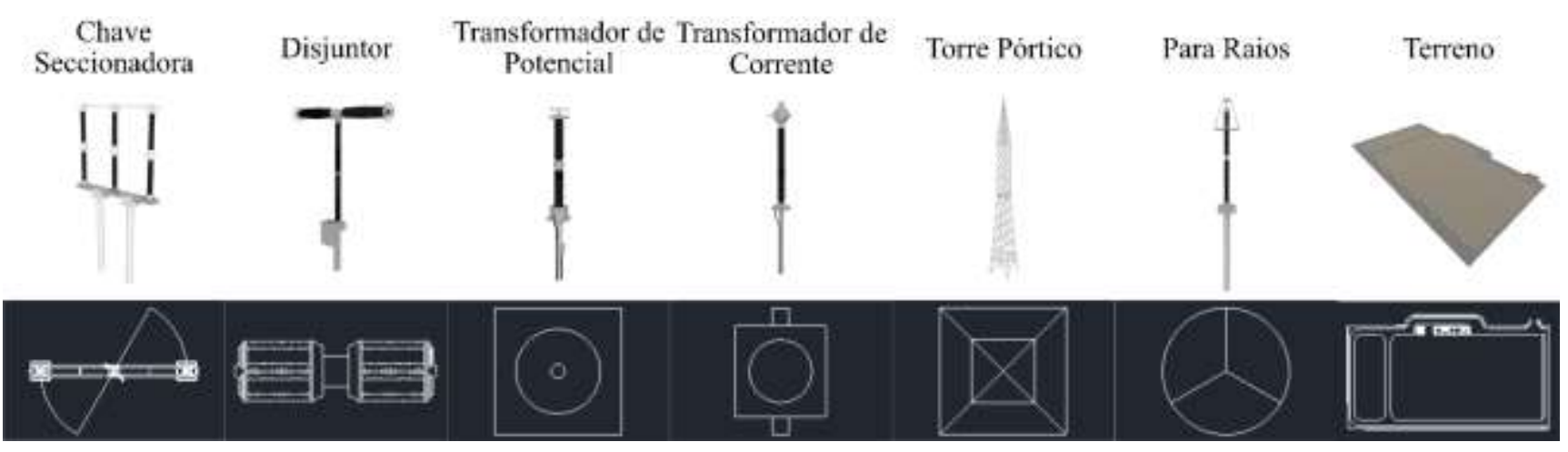

Fonte: Autores. 
Todos os modelos 3D foram registrados no GI referenciando sua classe, tensão, fabricante, fotografias e o símbolo CAD que os representem. Posteriormente acessados pelo ECAD e inseridos no desenho da planta baixa, formando o desenho CAD da SEE.

\subsection{Resultados do Processo de Integração}

A Figura 8 exemplifica a estrutura de dados do arquivo de integração no formato definido pelo metamodelo. O diagrama apresenta as informações do cenário, que inclui uma lista de modelos 3D e de condutores contendo os metadados fundamentais para reconstruir o AV em RV e identificar cada elemento.

Figura 8. Estrutura de dados do arquivo de integração entre ECAD e SRV.

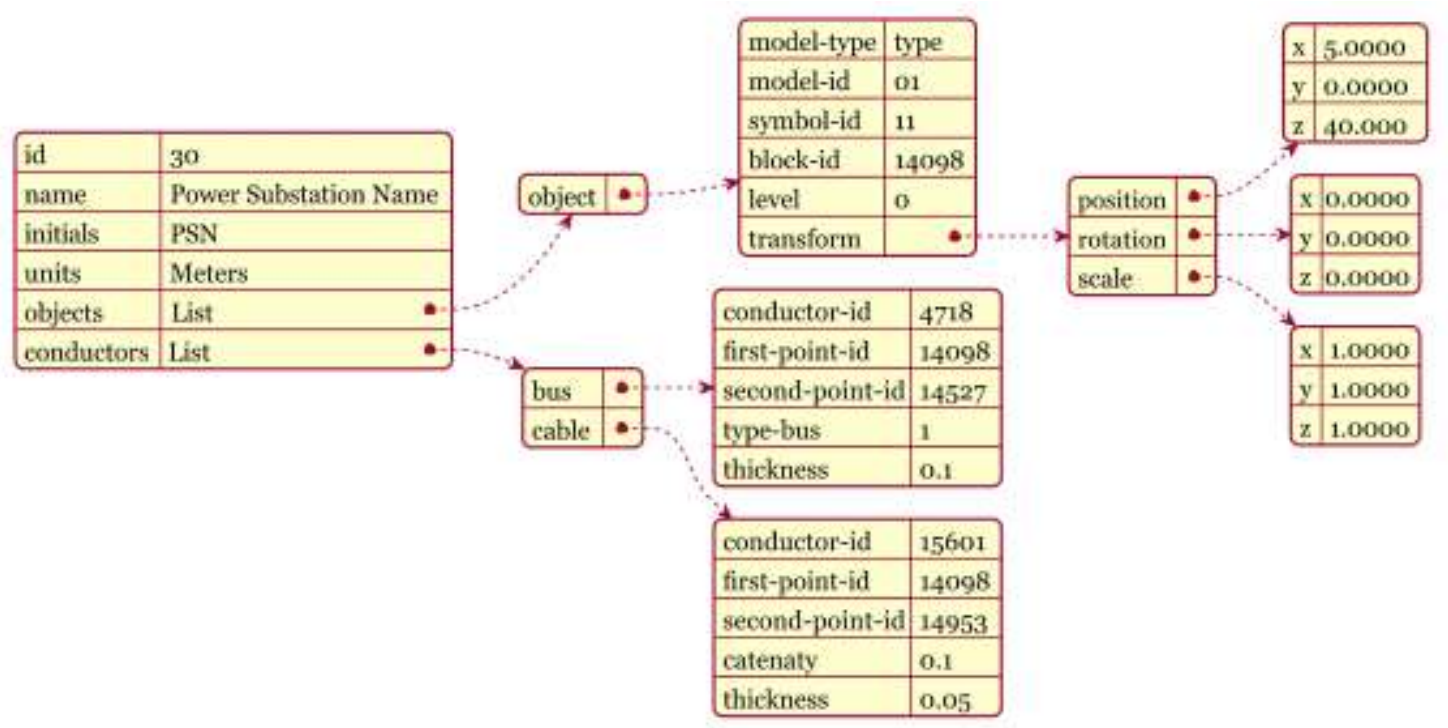

Fonte: Autores.

Nesse sentido, o SRV realizou a reconstrução do AV através do arquivo de integração da SEE solicitado ao GI. Logo após a requisição e recebimento dos dados, o módulo de reconstrução deu início a concepção do AV. Por meio dos metadados e dados de topologia do cenário os elementos foram posicionados e as conexões para condutores elétricos entre equipamentos foram desenhadas. O tempo de processamento para esse AV foi em média 9 segundos. A Figura 9 apresenta o AV da SEE concebida no SRV.

A Figura 10 apresenta os destaques dos condutores elétricos conectados entre equipamentos, barramentos e equipamentos supervisionados, neste sentido as demarcações em vermelho representam os cabos maleáveis, que podem conectar equipamentos ou barramentos aéreos. Em alternativa, as demarcações em azul claro representam os condutores rígidos, utilizados em barramentos próximos ao solo e que conectam muitos arranjos em uma linha de transmissão. Por fim, os destaques em amarelo representam equipamentos elegíveis à supervisão por meio de Supervisory Control and Data Acquisition (SCADA) conectado ao software de RV.

O tempo de integração e reconstrução dos AVs no SRV é um aspecto muito importante para a usabilidade do sistema. A quantidade de elementos que compõe o cenário influencia no tempo de reconstrução, nesse sentido, foram elaborados desenhos em plantas baixas com grupos de 60, 240, 540, 960, 1500 e 2160 modelos 3D, que representam a média de elementos das SEEs da companhia de energia elétrica. Os modelos 3D anteriores se reiteram a medida que novos modelos são adicionados, garantindo a constância nos grupos posteriores. 
Research, Society and Development, v. 10, n. 14, e221101421765, 2021

(CC BY 4.0) | ISSN 2525-3409 | DOI: http://dx.doi.org/10.33448/rsd-v10i14.21765

Figura 9. Subestação de energia elétrica integrada ao SRV.

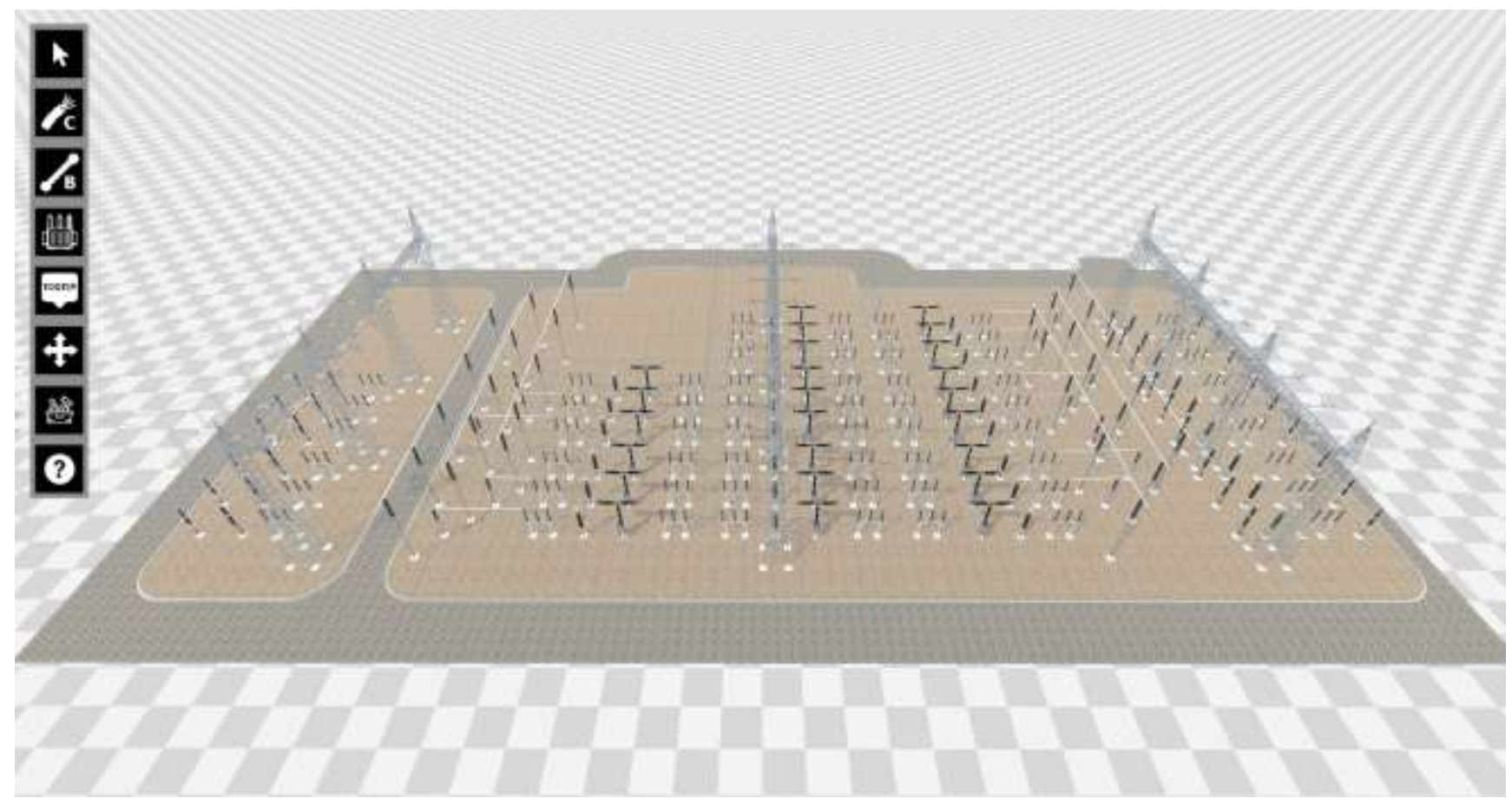

Fonte: Autores.

Figura 10. Destaque dos condutores conectados entre equipamentos, onde o destaque é em vermelho representa os cabeamentos maleáveis e em verde os barramentos rígidos.

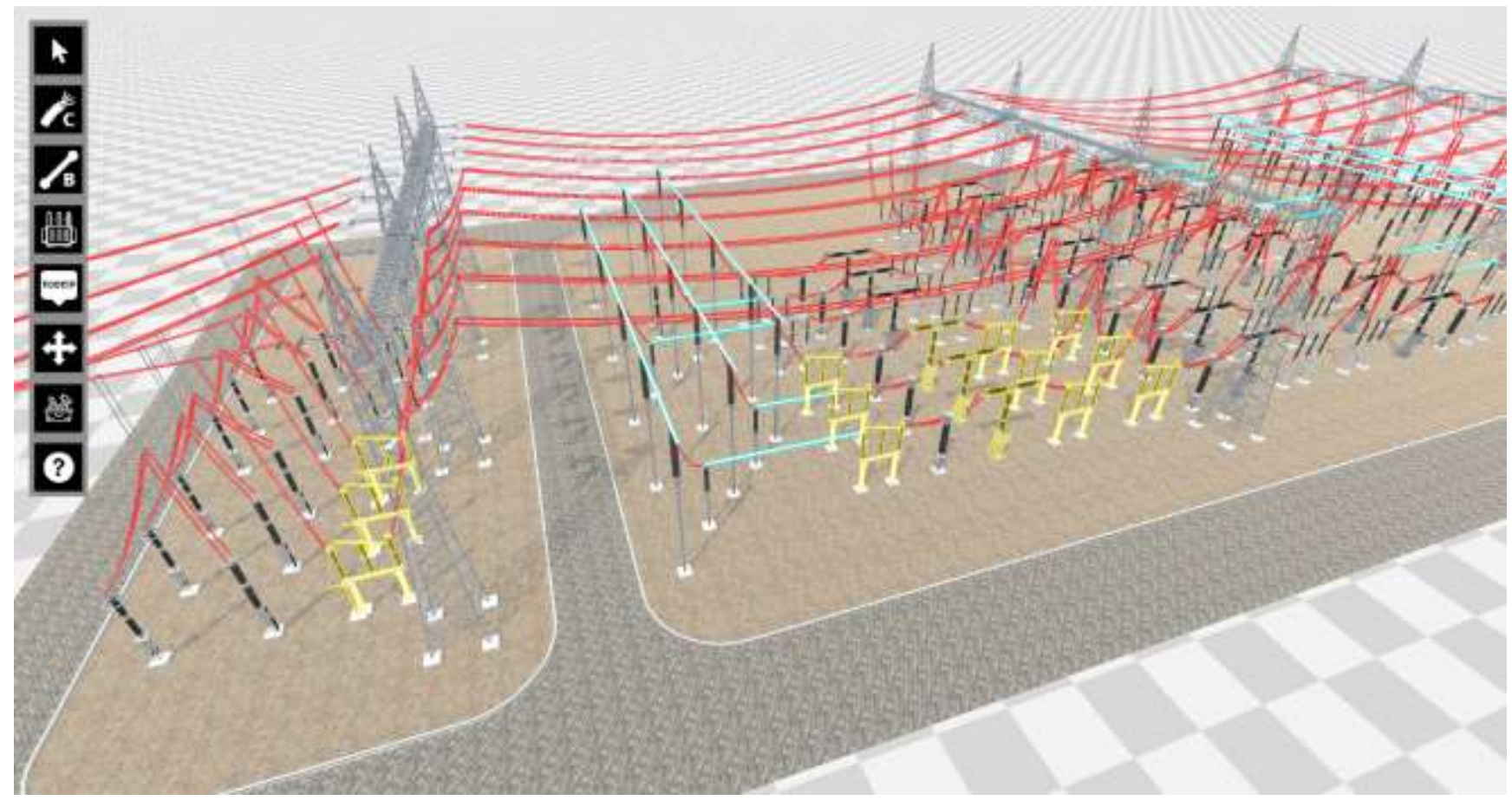

Fonte: Autores.

Os testes foram realizados através de cinco computadores com configurações de hardware e software compatíveis 
com empresas, escolas e residências:

- Computador 1: processador i7 8750H, placa gráfica GeForce 1060 Max-Q, memória de 16gb e sistema operacional Windows 10 Home;

- Computador 2: processador i5 7400, placa gráfica GeForce 1070, memória de 16gb e sistema operacional Windows 10 Home;

- Computador 3: processador i7 7490, placa gráfica Radeon R7 370, memória de 8gb e sistema operacional Windows 10 Home;

- Computador 4: processador i7 6700, placa gráfica GeForce GT420, memória de 8gb e sistema operacional Windows 10 Home;

- Computador 5: processador i3 370M, placa gráfica Intel HD Graphics, memória de 3gb e sistema operacional Windows 10 Home.

A Figura 11 apresenta o gráfico com as amostras de tempo em que cada computador concluiu o processo de integração e reconstrução.

Figura 11. Gráfico de desempenho de integração e reconstrução 3D no SRV.

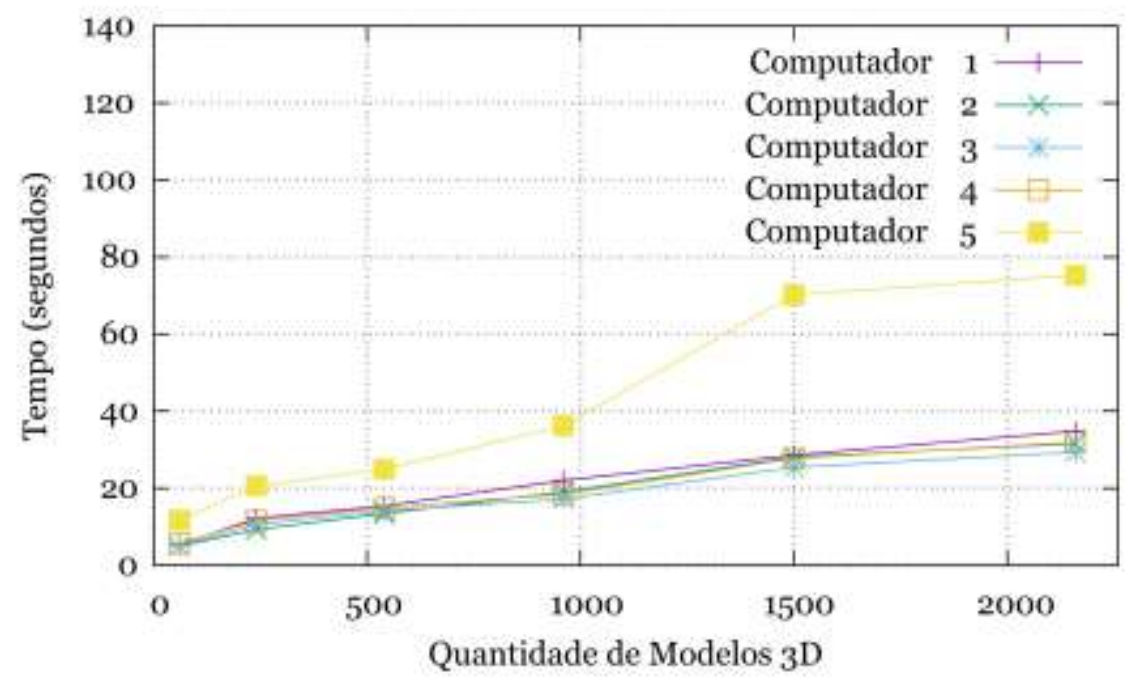

Fonte: Autores.

\section{Discussão e Conclusões}

Este trabalho propôs uma metodologia de integração entre plantas baixas CAD e SRV através de estruturas de dados geradas por uma definição de metamodelo. Uma arquitetura de software para gerenciar a integração foi apresentada. A avaliação da proposta foi realizada através de testes de desempenho de integração e reconstrução através de um estudo de caso aplicado ao desenvolvimento de SEEs de um projeto de P\&D.

O processo de integração, coordenado pelo Gerenciador de Integração, foi um importante recurso que possibilitou a atualização simultânea via internet de todos os SRV conectados a ele. A arquitetura de software adotou o conceito clienteservidor e permitiu, através de serviços de rede REST, o acesso de aplicações externas para consumo dos recursos, como acesso à biblioteca de modelos 3D e de símbolos. Desta forma, várias instâncias do ECAD e do SRV puderam acessar de forma simultânea o desenho de engenharia em planta baixa e o cenário 3D no SRV.

O tempo de integração da SE desenvolvida neste trabalho foi de sete segundos, um tempo aceitável para que o SRV 
forneça ao usuário um $\mathrm{AV}$ completo para navegação e interação. O tamanho da SE e a quantidade de elementos são compatíveis com grande parte das SEs da companhia de energia elétrica. Além do tempo de integração, o Ambiente Virtual foi replicado exatamente como desenhado em planta baixa, respeitando o posicionamento e orientação dos elementos.

Através de testes, pôde-se observar o desempenho do SI. Os testes de desempenho para cenários com quantidades de $60,240,540,960,1500$ e 2160 elementos se demonstraram adequados aos limites de tempo. As amostras de tempo foram em média $6.46 \mathrm{~s}, 12.82 \mathrm{~s}, 16.54 \mathrm{~s}, 22.54 \mathrm{~s}, 35.94 \mathrm{~s}$ e $40.56 \mathrm{~s}$ respectivamente, considerando o baixo desempenho do computador 5 que possui uma configuração de hardware inferior aos demais computadores, evidenciado na Figura 9. Neste sentido, pode-se destacar que a configuração do hardware dos computadores é o principal influenciador no desempenho da integração.

Através da análise dos trabalhos correlatos, a Tabela 2 foi elaborada apresentando as características em comum com este trabalho. Sendo assim, são evidenciadas as principais contribuições no contexto científico.

Tabela 2. Tabela comparativa das características utilizada neste trabalho e nos trabalhos correlatos.

\begin{tabular}{lllllll}
\hline Trabalho & CAD & Integração & Metamodelo & Grafos & Topologia & RTE \\
\hline Han et al. (2019) & 3D & Automático & Não & Não & Não & Sim \\
\hline Freeman et al. (2016) & 3D & Automático & Não & Não & Não & Sim \\
\hline Lorenz et al. (2016) & 3D & Automático & Não & Não & Não & Sim \\
\hline Martin et al. (2017) & 3D & Automático & Sim & Não & Não & Não \\
\hline Mattioli (2015) & 2D & Semiautomático & Não & Não & Não & Não \\
\hline Zawadzki et al. (2018) & 3D & Semiautomático & Não & Não & Não & Não \\
\hline Este trabalho & 2D & Automático & Sim & Sim & Sim & Sim \\
\hline
\end{tabular}

Fonte: Autores.

Em trabalhos futuros, deseja-se aprimorar o SI para uso de modelos paramétricos e Building Information Modeling (BIM) para expandir as possibilidades de aplicação do Editor CAD e o sistemas de RV. Desta forma, se espera que a manipulação de plantas baixas CAD com representação simbólica, facilite o processo de concepção de Ambientes Virtuais para propósitos de revisão de projetos e simulações.

\section{Agradecimentos}

O presente trabalho foi realizado com apoio da Coordenação de Aperfeiçoamento de Pessoal de Nível Superior Brasil (CAPES) - Código de Financiamento 001, pela Companhia Energética de Minas Gerais (CEMIG) e Agência Nacional de Energia Elétrica (ANEEL) por meio do P\&D GT0618, a quem os autores agradecem.

\section{Referências}

Baca, M. \& Getty Research Institute (Orgs.). (2008). Introduction to metadata (2nd ed). Getty Research Institute.

Ciprian Firu, A., Ion Tapîrdea, A., Ioana Feier, A., \& Drăghici, G. (2021). Virtual reality in the automotive field in industry 4.0. Materials Today: Proceedings, 45, 4177-4182. https://doi.org/10.1016/j.matpr.2020.12.037

Freeman, I. J., Salmon, J. L., \& Coburn, J. Q. (2016). Cad integration in virtual reality design reviews for improved engineering model interaction. Volume 11: Systems, Design, and Complexity, V011T15A006. https://doi.org/10.1115/IMECE2016-66948

Guedes, G. T. A. (2012). Um metamodelo UML para a modelagem de requisitos em projetos de sistemas multiagentes. https://lume.ufrgs.br/handle/10183/56847 
Han, Y.-S., Lee, J., Lee, J., Lee, W., \& Lee, K. (2019). 3D CAD data extraction and conversion for application of augmented/virtual reality to the construction of ships and offshore structures. International Journal of Computer Integrated Manufacturing, 32(7), 658-668. https://doi.org/10.1080/0951192X.2019.1599440

ISO. (2020). Information Technology for Learning, Education and Training — Human Factor Guidelines for Virtual Reality Content — Part 1: Considerations when Using VR Content; Standard ISO/IEC TR 23842-1:2020; International Organization for Standardization: Geneva, Switzerland.

Kovar, J., Mouralova, K., Ksica, F., Kroupa, J., Andrs, O., \& Hadba, Z. (2016). Virtual reality in context of Industry 4.0 proposed projects at Brno University of Technology. 2016 17th International Conference on Mechatronics - Mechatronika (ME), 22, 1-7.

Kuts, V.; Otto, T.; Caldarola, E, G.; Modoni, G. E.; Sacco, M. (2018). Enabling the Teaching Factory leveraging a Virtual Reality system based on the Digital Twin. In: K. Helin, M. Poyade, M. D'Cruz, R. Eastgate (Ed.). The Industrial Track of EuroVR 2018: Proceedings of the 15th Annual EuroVR Conference (26-31). VTT Technical Research Centre of Finland.

Lorenz, M., Spranger, M., Riedel, T., Pürzel, F., Wittstock, V., \& Klimant, P. (2016). Cad to vr - a methodology for the automated conversion of kinematic cad models to virtual reality. Procedia CIRP, 41, 358-363. https://doi.org/10.1016/j.procir.2015.12.115

Martin, G., Steger, W., Stelzer, R., \& Bertelmann, K. (2017). Meta-model for VR-based design reviews. 4, $337-346$.

Mattioli, L. (2015). Uma proposta de um procedimento para a geração semiautomática de ambientes virtuais para subestações de energia elétrica [Universidade Federal de Uberlândia]. https://doi.org/10.14393/ufu.di.2015.338

Nugraha Bahar, Y., Landrieu, J., Pére, C., \& Nicolle, C. (2014). CAD data workflow toward the thermal simulation and visualization in virtual reality. International Journal on Interactive Design and Manufacturing (IJIDeM), 8(4), 283-292. https://doi.org/10.1007/s12008-013-0200-5

Pessoa, A.; Gomes, D., Jr.; Reis, P.; Paiva, A.; Silva, A.; Braz, G.; Araújo, A. (2017). Uma ferramenta de autoria para construção de ambientes de realidade virtual para subestações de energia baseada em panoramas aumentados. In: Conference on Graphics, Patterns and Images, 30. (SIBGRAPI), Niterói, RJ. Proceedings. Porto Alegre: Sociedade Brasileira de Computação.

Quevedo, W. X., Sánchez, J. S., Arteaga, O., Álvarez V., M., Zambrano, V. D., Sánchez, C. R., \& Andaluz, V. H. (2017). Virtual reality system for training in automotive mechanics. Augmented Reality, Virtual Reality, and Computer Graphics (Vol. 10324, p. 185-198). Springer International Publishing. https://doi.org/10.1007/978-3-319-60922-5_14

Roldán, J. J., Crespo, E., Martín-Barrio, A., Peña-Tapia, E., \& Barrientos, A. (2019). A training system for Industry 4.0 operators in complex assemblies based on virtual reality and process mining. Robotics and Computer-Integrated Manufacturing, 59, 305-316. https://doi.org/10.1016/j.rcim.2019.05.004

Sheridan, T. B. (2000). Interaction, imagination and immersion some research needs. Proceedings of the ACM Symposium on Virtual Reality Software and Technology - VRST '00, 1. https://doi.org/10.1145/502390.502392

Stark, R., Israel, J., \& Wöhler, T. (2010). Towards hybrid modelling environments-Merging desktop-CAD and virtual reality-technologies. CIRP Annals, 59(1), 179-182. https://doi.org/10.1016/j.cirp.2010.03.102

Technologies, U. ([s.d.]). Unity real-time development platform. https://unity.com/

Tori, R., Kirner, C., \& Siscoutto, R. A. (2006). Fundamentos e tecnologia de realidade virtual e aumentada. Editora SBC.

Wolfartsberger, J. (2019). Analyzing the potential of Virtual Reality for engineering design review. Automation in Construction, 104, 27-37. https://doi.org/10.1016/j.autcon.2019.03.018

Zawadzki, P., Gorski, F., Bun, P., Wichniarek, R., \& Szalanska, K. (2018). Virtual reality and cad systems integration for quick product variant design. Advances in Manufacturing (p. 599-608). Springer International Publishing. https://doi.org/10.1007/978-3-319-68619-6_57 